\title{
Renal Cell Carcinoma, Unclassified with Medullary Phenotype and Synchronous Renal Clear Cell Carcinoma Present in a Patient with No Sickle Cell Trait/Disease: Diagnostic and Therapeutic Challenges
}

\author{
JENNY Z. LAI ${ }^{1,2,3}$, H. HENRY LAI ${ }^{2}$ and DENGFENG CAO ${ }^{3}$ \\ ${ }^{1}$ University College, Washington University in St. Louis, St. Louis, MO, U.S.A.; \\ ${ }^{2}$ Division of Urologic Surgery, Department of Surgery, School of Medicine, \\ Washington University in Saint Louis, St. Louis, MO, U.S.A.; \\ ${ }^{3}$ Department of Pathology and Immunology, School of Medicine, \\ Washington University in Saint Louis, St. Louis, MO, U.S.A.
}

\begin{abstract}
Renal medullary carcinoma (RMC) is an aggressive high-grade renal cell carcinoma (RCC) associated almost exclusively with sickle cell trait or sickle cell disease. However, RCC with RMC features has rarely been reported in patients with no sickle cell trait or disease. Renal cell carcinoma unclassified with medullary phenotype (RCCU-MP) is a newly-coined term used by an international panel of experts to describe renal cell carcinoma showing morphologic and immunohistochemical features of renal medullary carcinoma in patients without sickle cell trait/disease. So far, only one study in the English literature has described five such cases. Here, we report a case with unique clinical and pathological features in a 76-year-old male patient without sickle cell trait. The patient had a history of colon cancer with liver and lung metastases and was found to have a new renal mass in his right kidney during the follow up. A right nephrectomy was performed and showed two separate masses (tumor 1 and tumor 2). Tumor 1 had histologic features of RMC and the tumor cells were positive for CK7, Pax8, and OCT4 and showed loss of nuclear INII expression. Tumor 1 was diagnosed as RCCUMP (6.3 cm, pT3aNx, WHO/ISUP nuclear grade 3). Tumor 2 showed features of clear cell type of RCC $(0.6 \mathrm{~cm}, p T l a N x$,
\end{abstract}

Correspondence to: Dengfeng Cao, MD, Ph.D., Department of Pathology and Immunology, Washington University School of Medicine, 660 S Euclid Ave Campus Box 8118, St. Louis, MO 63110, U.S.A. Tel: +1 3147475007, Fax: +1 3143628950, e-mail: dengfengcao@wustl.edu

Key Words: Kidney, renal cell carcinoma, medullary phenotype, diagnosis, prognosis.
WHO/ISUP grade 2) with intact nuclear INII expression. Three-months post-nephrectomy, the patient developed lung metastasis of RCCU-MP. To the best of our knowledge, this was the first documented case with synchronous RCCU-MP and clear cell RCC presenting in a patient without sickle cell trait. Careful histologic assessment with a panel of immunohistochemical biomarkers was helpful to render a correct diagnosis for early aggressive treatment.

Renal medullary carcinoma (RMC) is an aggressive highgrade renal cell carcinoma (RCC) associated almost exclusively with sickle cell trait or sickle cell disease (1). $\mathrm{RMC}$ has been determined to reproducibly harbor alterations in the SMARCBI/INII gene and loss of INI expression by immunohistochemistry, and these features have been proven to be a useful adjunct for the diagnosis of RMC (2). However, RCC with RMC histological features and loss of INI1 presenting in patients with no sickle cell trait has been rarely reported (2). Renal cell carcinoma, unclassified with medullary phenotype (RCCU-MP) is a newly-coined term for such tumor (3). So far, only one study has described five cases of RCCU-MP in patients without sickle cell trait. Here, we report a new case with unique clinical and pathological features in a 76-year-old male patient without sickle cell trait/disease. The patient had a history of colonic adenocarcinoma with liver metastasis and was found to have a new renal mass in his right kidney during the follow-up. Right nephrectomy showed two separate renal tumors including one with histologic features of RMC and loss of nuclear expression INI1 and the other with features of typical clear cell RCC and intact INI1 expression. To the best of our knowledge, this is the first documented case with synchronous RCCU-MP and clear cell RCC presenting in a 
patient without sickle cell trait/disease. The pathogenesis, diagnostic and therapeutic challenges are discussed.

\section{Case Presentation}

The patient was a 76-year-old Caucasian male with a newly identified renal mass in his right kidney during his regular follow-up for colon cancer with liver and lung metastases. His past medical history was significant for his metastatic sigmoid colon cancer that was subsequently cured. He initially presented with hematochezia in July 2009 and was found to have a stage III colonic adenocarcinoma which was resected (surgery performed on $7 / 2 / 2009$, tumor size $5.5 \mathrm{~cm}$, 3 of 13 lymph nodes with metastasis, pathologic stage pT3N1). He declined participation in clinical trial but proceeded with standard 6 months of FOLFOX adjuvant therapy. An elevated CEA was noted in August 2012, and CT scans showed a lesion in the liver, which was treated with radiofrequency ablation in January 2013. A dysplastic polyp was found in the fall 2013, which led to a completion colectomy in November 2013. There was no evidence of residual cancer in the removed specimen. Additional lesion in the liver was noted in January 2014, which was treated with cryotherapy. A lesion in his lung was treated with stereotactic radiation therapy in 2016. A more recent CT scan in late 2017 showed growth of a $7-\mathrm{cm}$ renal lesion (Figure 1A) in his right kidney. No significant lesions were identified in his lung and liver. His lab work-up was unremarkable. He was an Italian descent with no evidence or diagnosis of sickle cell trait or sickle cell disease. Through discussion in the multidisciplinary tumor board, the patient underwent a right laparoscopic nephrectomy and the specimen was submitted for pathology evaluation.

Grossly, the right radical nephrectomy specimen weighed 1197.7 grams and measured $14.4 \times 8.7 \times 6.8 \mathrm{~cm}$. It was a $14.5 \times 7.1 \times 7.1 \mathrm{~cm}$ kidney containing a $6.3 \times 4.8 \times 4.0 \mathrm{~cm}$ white tan, focally hemorrhagic, soft and ill-defined mass (tumor 1) that was located in the mid pole and grossly invaded the renal pelvis and renal sinus adipose tissue. The closest renal artery margin was $2.5 \mathrm{~cm}$ from tumor 1 . A separate $1.0 \times 0.7 \times 0.5 \mathrm{~cm}$ soft tan and ill-defined mass (tumor 2) was also identified in the superior pole of the same kidney. Tumor 2 was $0.5 \mathrm{~cm}$ from and separate from tumor 1 . Representative sections from both masses were submitted for microscopic evaluation. Histologically, tumor 1 and tumor 2 had different morphology. The tumor cells in tumor 1 formed nests/sheets, focally single glands and cribriform glands with cystic changes in a background of desmoplastic stroma suggestive of poorly differentiated adenocarcinoma (Figure 1B). There was acute inflammation within the tumor nests/glands and stroma. The tumor cells had vesicular nuclei and prominent nucleoli (ISUP/WHO nuclear grade 3). Mitotic figures were frequently seen (Figure 1C). In contrast to tumor 1, the tumor cells from tumor 2 showed a typical morphology of conventional clear cell renal cell carcinoma with ISUP/WHO nuclear grade 2 (Figure 1D). Immunohistochemical stains were performed on both tumors. The tumor cells in the tumor 1 were positive for CK7, CK-34BE12, PAX8, focally positive for OCT4, with loss of nuclear INI1 (Figure 2), and negative for CK20, CDX2, GATA3, P40, P63, RCC, CD10, TTF1, Napsin A, CD117, Her2, and thyroglobulin. Mucicarmine stain highlighted intraluminal mucin. Overall the morphology and immunoprofile of tumor 1 supported the diagnosis of renal cell carcinoma, unclassified with medullary phenotype. The tumor cells in the tumor 2 had an intact immunohistochemical expression of nuclear INI1 and was positive for RCC and CD10 and negative for CK7 (Data not shown). The final pathology diagnosis was right kidney with synchronous RCCU-MP $(6.3 \mathrm{~cm}, \mathrm{pT} 3 \mathrm{aNx}, \mathrm{WHO} / \mathrm{ISUP}$ nuclear grade 3) and separate clear cell RCC $(0.6 \mathrm{~cm}$, pT1aNx, WHO/ISUP nuclear grade 2).

Postoperatively, the patient recovered very well from his right nephrectomy. On the three-month follow-up, CT scans showed a stable aortic aneurysm and multiple new pulmonary nodules seen throughout the lungs (Figure 3A). Of note the largest nodule within the right upper lobe was located medially and may be amenable to bronchoscopy. An endobronchial ultrasound guided fluoroscopy assisted fine needle aspiration was performed. The direct smears (Figure 3B left) showed groups of tumor cells with rhabdoid cytomorphologic features with ample eosinophilic and occasionally vacuolated cytoplasm, eccentric nuclei with irregular nuclear contours and nuclear pleomorphism and prominent nucleoli. The cell block showed high-grade tumor cells with solid sheets arrangement and neutrophilic infiltrate. The tumor cell was positive for CK7 and PAX8, and negative for CK20. The cytological features and immuno-profile support the diagnosis of metastasis of renal cell carcinoma, unclassified with medullary phenotype. No metastasis of clear cell RCC or colon cancer was identified. The patient is currently on Afinitor $10 \mathrm{mg}$ daily, avastin and C1D1.

\section{Discussion}

RMC is a highly aggressive renal cell carcinoma arising in the collecting duct system and the diagnosis requires careful correlation with the status of sickle cell trait, and less frequently, with sickle cell disease. A panel of international experts has recently proposed a provisional diagnostic terminology, renal cell carcinoma, unclassified, with medullary phenotype (RCCU-MP), based on the encounter with an extraordinarily rare tumor with RMC morphology and immuno-phenotype in an individual proven not to have a hemoglobinopathy of sickle cell trait or disease $(2,3)$. So far, there is only one study with five cases of RCCU-MP reported by Sirohi et al. in patients without sickle cell trait 

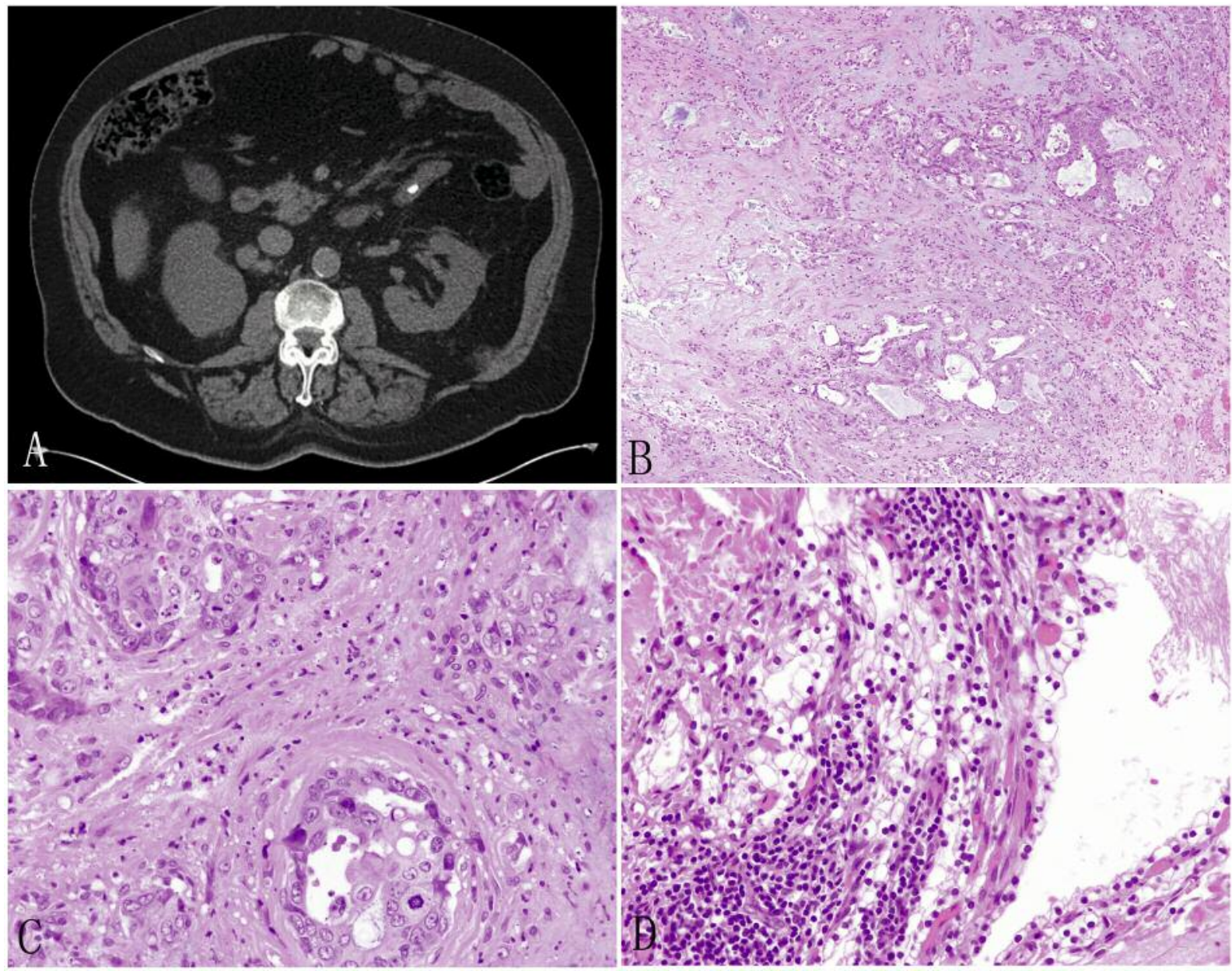

Figure 1. Synchronous Renal cell carcinoma (RCC), unclassified with medullary phenotype (RCCU-MP) and a separate clear cell type RCC. A: $C T$ scan showing right renal mass (RCCU-MP); B-C: Histologically, the RCCU-MP shows features of poorly differentiated adenocarcinoma with cystic changes, neutrophil infiltrate in the tumor cells and desmoplastic stroma, grade 3 nuclei and mitotic figures; D: The tumor cells of the clear cell RCC shows clear cytoplasm and grade 2 nuclei (H\&E stain, B, ×100; C-D, x400).

(2). They found that the five cases of RCCU-MP were morphologically indistinguishable from RMC, but lack evidence of sickle cell trait or disease. We report the sixth case of RCCU-MP with more unique features than the fivereported cases. The unique features in our case included i), onset at the oldest age (76-year-old); ii), many years of history of sigmoid colon cancer with metastasis to liver and lung; and iii), coexistences of a synchronous clear cell RCC. These features make the diagnostic and therapeutic process more challenging.

RMCs typically present in young patients in their third decade, with reported age range between 5 to 69 years $(4,5)$. The oldest case was described in a 69-year-old individual (5). RMCs show a strong male predilection with a male-to-female ratio of $\sim 2: 1$ and arise more frequently in the right kidney. The recently reported five cases of RCCU-MP by Sirohi et al. included four males and one female with an age range of 24 to 71 years (2). So far, our case had the oldest age (76 years) and also arose from the right kidney. Cytogenetic and molecular findings have been reported on the pathogenesis of RMCs. RMCs harbor alternations in the SMARCBI/INII gene, including loss of heterozygosity (6), hemizygous deletions (7), and even loss of chromosome 22 (4). Very recent data have also identified balanced translocations involving the SMARCB1 locus in 4 of 4 tested cases of RMC (8), a mechanism that may explain the prior observations of hemizygous loss of SMARCBI in these tumors (2).

Histological features of RMC and RCCU-MP show highgrade infiltrative adenocarcinomas, frequently with reticular or microcystic patterns reminiscent of yolk sac tumor, and cribriform, adenoid cystic-like, solid, or trabecular architecture eliciting a desmoplastic stromal response (9). 


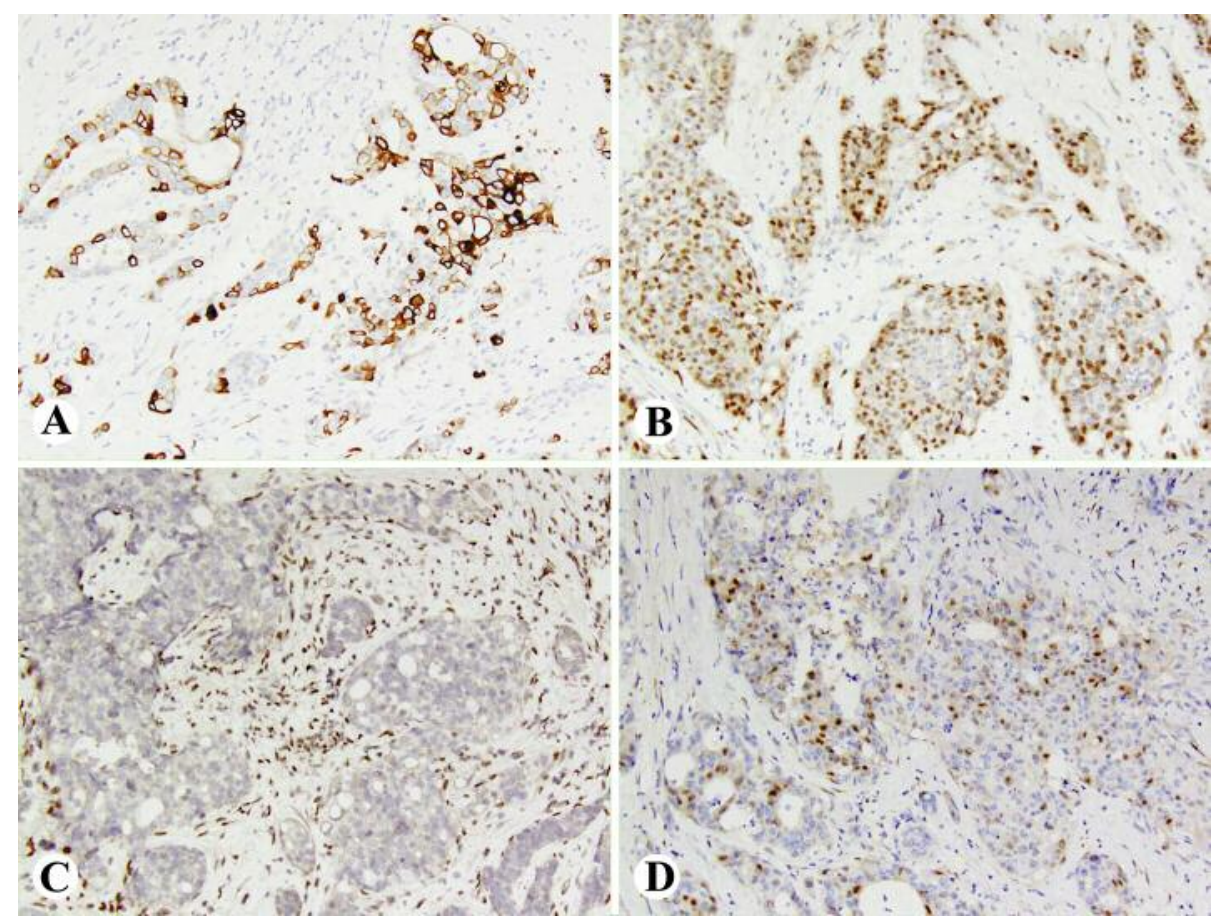

Figure 2. Immunohistochemistry of the RCCU-MP. The tumor cells are positive for CK7 (A), PAX8 (B), negative for nuclear INI1 (C) and focallypositive for OCT4 (D) $(A-D, \times 200)$.
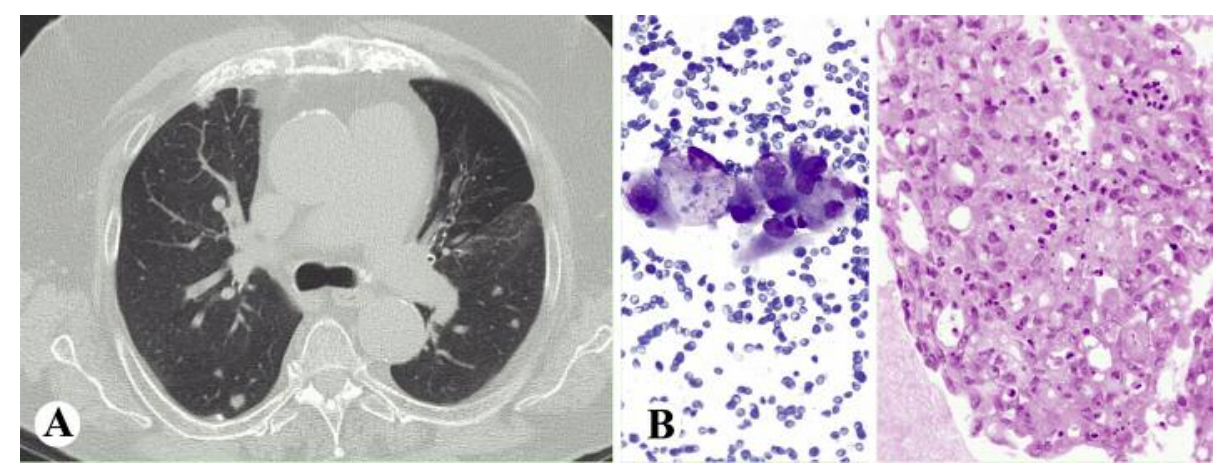

Figure 3. Lung with RCCU-MP metastasis. A: CT scan showing multiple enhanced lung nodules; B: Endobronchial ultrasound-guided fine needle aspiration showing groups of atypical rhabdoid tumor cells on direct smears (left, Diff Quick, x600) and solid arrangement of tumor cells with neutrophils infiltrate in the cell block (right, $H \& E$ stain, $\times 400$ ).

RMCs often show areas with rhabdoid cytomorphology, although poorly differentiated solid areas are quite prevalent and stromal inflammation is common. The present case showed the typical histologic features of RMC or RCCUMP. The reported five RCCU-MP cases showed loss of nuclear INI1 (5/5), positive immunoreactivity for OCT3/4 (4/5), CK7 (3/4) and PAX8 (5/5) (2). The present case showed similar histologic features and same immuno profile as the five reported cases. In addition, within the same nephrectomy specimen, a synchronous clear cell type of RCC was also found and showed striking differences of the morphology and immuno profile to the RCCU-MP.

RMCs and RCCU-MP are aggressive tumors, frequently with metastatic lesions at the time of presentation and dismal outcomes, with the longest reported survival of 15 months (average 15 weeks) (4). The available follow-up data of the 
five reported RCCU-MP cases showed that 4 patients died at $3,3,9$, and 27 months. In the present case, the patient was identified to have multiple new lung nodules on the three months follow up. The endobronchial ultrasound-guided fine needle aspiration shows the tumor cells with rhabdoid cytological features and immuno profile of RCCU-MP. No metastasis of clear cell RCC or prior colon cancer components are identified although the patient has the history of colon cancer metastasis to lung and liver. The patient is currently doing well with Afinitor, Avastin and supportive treatment. Recently nivolumab, an anti-programmed cell death 1 (PD-1) monoclonal antibody, has been approved for treating metastatic renal cell carcinoma (10) and it is unknown whether this drug is effective for metastatic RCCU-MP or not.

In summary, renal cell carcinoma, unclassified with medullary phenotype (RCCU-MP) is a newly-described and rarely reported entity that can be seen in the patients without sickle cell trait/disease. We report the oldest patient with this entity and coexistence with a separate clear cell type of renal cell carcinoma presenting in a patient with history of sigmoid colon adenocarcinoma with liver and lung metastases. RCCU-MP is a very aggressive RCC. On the three-month follow-up post-nephrectomy, the patient in this report was found to have lung metastasis of the RCCU-MP. Careful histological assessment with immunostainings of a panel of biomarkers was helpful to render a correct diagnosis for early aggressive treatment.

\section{References}

1 Davis CJ Jr., Mostofi FK and Sesterhenn IA: Renal medullary carcinoma. The seventh sickle cell nephropathy. Am J Surg Pathol 19: 1-11, 1995.

2 Sirohi D, Smith SC, Ohe C, Colombo P, Divatia M, Dragoescu E, Rao P, Hirsch MS, Chen YB, Mehra R and Amin MB: Renal cell carcinoma, unclassified with medullary phenotype: poorly differentiated adenocarcinomas overlapping with renal medullary carcinoma. Hum Pathol 67: 134-145, 2017.
3 Amin MB, Smith SC, Agaimy A, Argani P, Compérat EM, Delahunt B, Epstein JI, Eble JN, Grignon DJ, Hartmann A, Hes O, Hirsch MS, Jimenez RE, Kunju LP, Martignoni G, McKenney JK, Moch H, Montironi R, Paner GP, Rao P, Srigley JR, Tickoo SK and Reuter VE: Collecting duct carcinoma versus renal medullary carcinoma: an appeal for nosologic and biological clarity. Am J Surg Pathol 38: 871-874, 2014.

4 Swartz, MA, Karth, J, Schneider, DT, Rodrguez R, Beckwith JB and Perlman EJ: Renal medullary carcinoma: clinical, pathologic, immunohistochemical, and genetic analysis with pathogenetic implications. Ped Urol 60: 1083-1089, 2002.

5 Watanabe IC, Billis A, Guimarães MS, Alvarenga M, de Matos AC, Cardinalli IA, Filippi RZ, de Castro MG and Suzigan S: Renal medullary carcinoma: report of seven cases from Brazil. Mod Pathol 20: 914-920, 2007.

6 Liu Q, Galli S, Srinivasan R, Linehan WM, Tsokos M and Merino MJ: Renal medullary carcinoma - molecular, immunohistochemistry, and morphologic correlation. Am J Surg Pathol 37: 368-374, 2013.

7 Calderaro J, Moroch J, Pierron G, Pedeutour F, Grison C, Maillé P, Soyeux P, de la Taille A, Couturier J, Vieillefond A, Rousselet MC, Delattre O and Allory Y: SMARCB1/ INI1 inactivation in renal medullary carcinoma. Histopathology 61: 428-435, 2012.

8 Calderaro J, Masliah-Planchon J, Richer W, Maillot L, Maille P, Mansuy L, Bastien C, de la Taille A, Boussion H, Charpy C, Jourdain A, Bléchet C, Pierron G, Gentien D, Choudat L, Tournigand C, Delattre O, Allory Y and Bourdeaut F: Balanced translocations disrupting SMARCB1 are hallmark recurrent genetic alterations in renal medullary carcinomas. Eur Urol 69: 1055-1061, 2016.

9 Gupta R, Billis A, Shah RB, Moch H, Osunkoya AO, Jochum W, Hes O, Bacchi CE, de Castro MG, Hansel DE, Zhou M, Vankalakunti M, Salles PG, Cabrera RA, Gown AM, and Amin MB: Carcinoma of the collecting ducts of Bellini and renal medullary carcinoma. Am J Surg Pathol 36: 1265-1278, 2012.

10 Kobari Y, Kondo T, Tagaki T, Ome K, Nakazawa H and Tanabe K: Rapid progressive disease after nivolumab therapy in three patients with metastatic renal cell carcinoma. In Vivo 31: 769-771, 2017.

Received April 7, 2018

Revised April 30, 2018

Accepted May 2, 2018 